\title{
MCMC for Generalized Linear Mixed Models with glmmBUGS
}

\author{
by Patrick Brown and Lutong Zhou
}

\begin{abstract}
The glmmBUGS package is a bridging tool between Generalized Linear Mixed Models (GLMMs) in R and the BUGS language. It provides a simple way of performing Bayesian inference using Markov Chain Monte Carlo (MCMC) methods, taking a model formula and data frame in R and writing a BUGS model file, data file, and initial values files. Functions are provided to reformat and summarize the BUGS results. A key aim of the package is to provide files and objects that can be modified prior to calling BUGS, giving users a platform for customizing and extending the models to accommodate a wide variety of analyses.
\end{abstract}

\section{Introduction}

There are two steps in model fitting that are much more time consuming in WinBUGS than in R. First, unbalanced multi-level data needs to be formatted in a way that BUGS can handle, including changing categorical variables to indicator variables. Second, a BUGS model file must be written. The glmmBUGS package addresses these issues by allowing users to specify models as formulas in $\mathrm{R}$, as they would in the $\mathrm{glm}$ function, and provides everything necessary for fitting the model with WinBUGS or OpenBUGS via the R2WinBUGS package (Sturtz et al., 2005).

The glmmBUGS package creates the necessary BUGS model file, starting value function, and suitably formatted data. Improved chain mixing is accomplished with a simple reparametrization and the use of sensible starting values. Functions are provided for formatting and summarizing the results. Although a variety of models can be implemented entirely within glmmBUGS, the package intends to provide a basic set of data and files for users to modify as necessary. This allows the full flexibility of BUGS model specification to be exploited, with much of the initial "grunt work" being taken care of by glmmBUGS.

\section{Examples}

\section{Independent random effects}

Consider the bacteria data from the MASS package:

\footnotetext{
$>$ library (MASS)

$>$ data (bacteria)

$>$ head(bacteria)
}

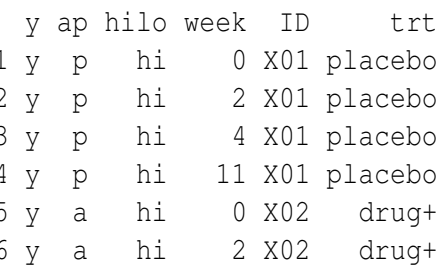

The variables to be considered are: $y$, the presence or absence of bacteria in a sample coded as " $y$ " and " $n$ " respectively; week, the time of the observation; ID, the subject identifier; and trt giving the treatment group as "placebo", "drug", or "drug+".

A generalized linear mixed model is applied to the data with:

$$
\begin{aligned}
Y_{i j} & \sim \operatorname{Bernoulli}\left(p_{i j}\right) \\
\operatorname{logit}\left(p_{i j}\right) & =\mu+x_{i j} \beta+V_{i} \\
V_{i} & \sim \text { iid } \mathrm{N}\left(0, \sigma^{2}\right)
\end{aligned}
$$

where: $Y_{i j}$ indicates the presence or absence of bacteria of the $i$ th person at week $j$; covariates $x_{i j}$ are week and indicator variables for treatment; $p_{i j}$ denotes the probability of bacteria presence; $V_{i}$ is the random effect for the $i$ th patient, assumed to be normal with mean 0 and variance $\sigma^{2}$. To improve the mixing of the Markov chain, a reparametrized model is fitted with:

$$
\begin{aligned}
Y_{i j} & \sim \operatorname{Bernoulli}\left(p_{i j}\right) \\
\operatorname{logit}\left(p_{i j}\right) & =R_{i}+w_{i j} \gamma \\
R_{i} & \sim \mathrm{N}\left(\mu+g_{i} \alpha, \sigma^{2}\right) .
\end{aligned}
$$

Here $g_{i}$ is the (indicator variable for) treatment group for subject $i$ and $w_{i j}$ is the week that observation $j$ was taken. Note that the two models are the same, with $V_{i}=R_{i}-\mu-g_{i} \alpha, \beta=(\gamma, \alpha)$ and $x_{i j}=\left(w_{i j}, g_{i}\right)$. The model in (1) has strong negative dependence between the posterior samples of $V_{i}$ and $\mu$, whereas $R_{i}$ and $\mu$ in (2) are largely independent.

As BUGS only allows numeric data, and cannot have the ' + ' sign in variable names, the data are recoded as follows:

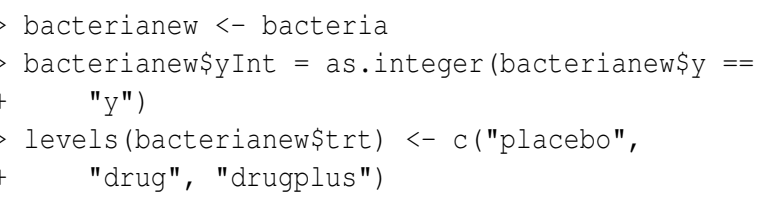

The primary function in the package is glmmBUGS, which does the preparatory work for fitting the model in (2) with:

$>$ library (glmmBUGS)

$>$ bacrag $<-$ glmmBUGS (formula = yInt 


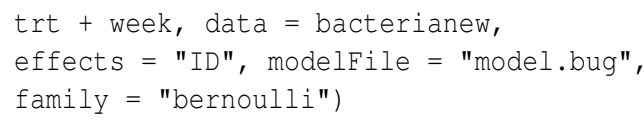

This specifies yInt as a Bernoulli-valued response, trt and week as fixed-effect covariates, and the "ID" column for the random effects. The result is a list with three elements:

ragged is a list containing the data to be passed to WinBUGS;

pql is the result from fitting the model with the glmmPQL function in MASS package.

startingValues is a list of starting values for the parameters and random effects, which is obtained from the glmmPQL result.

In addition, two files are written to the working directory

'model.bug' is the BUGS model file

'getInits. $\mathrm{R}^{\prime}$ contains the $\mathrm{R}$ code for a function to generate random starting values.

To accommodate unbalanced designs, the data are stored as ragged arrays, as described in the section "Handling unbalanced datasets" of the WinBUGS manual (Spiegelhalter et al., 2004). The ragged result has a vector element "SID" indicating the starting position of each individual's observations in the dataset. The covariates are split into elements "XID" and "Xobservations" for the individual-level and observation-level covariates respectively:

$>$ names (bacrag\$ragged)

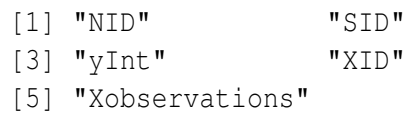

The model file consists of an outer loop over ID levels and an inner loop over observations. The details of how the model is implemented are best understood by examining the 'model.bug' file and consulting the WinBUGS manual.

At this stage the user is expected to modify the files and, if necessary, the data to refine the model, set appropriate priors, and ensure suitability of starting values. Before using the bugs function in the R2WinBUGS package to sample from the posterior, the starting value function get Inits must be prepared. First, the file 'getlnits.R' (which contains the source for getInits) should be edited and sourced into R. Next, the list containing the PQLderived starting values must be assigned the name startingValues, as getInits will be accessing this object every time it is run.

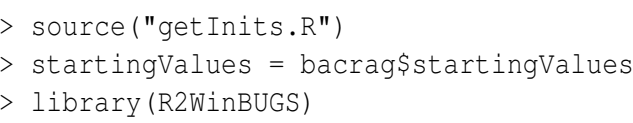

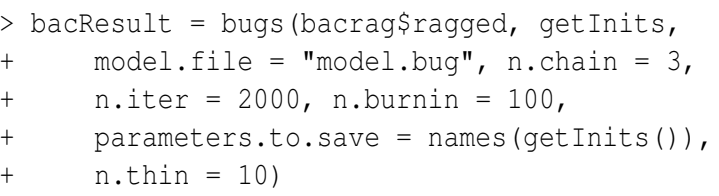

\section{Post WinBUGS commands}

After running WinBUGS, a series of functions in the glmmBUGS package can be used to manipulate and check the simulation results. The restoreParams function is used to restore the original parametrization from (1) and assign the original names to the group or subject identifiers.

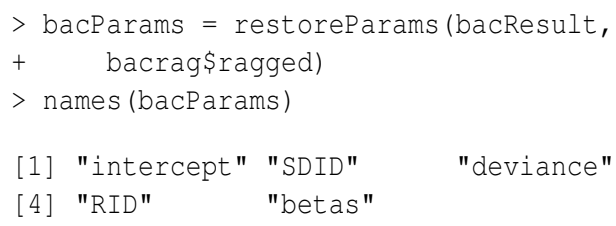

The result is a set of posterior samples for $\mu$ (intercept), $\sigma$ (SDID), all the $V_{i}$ (RID), and $\beta$ (betas). Posterior means and credible intervals are obtained with the summarychain function.

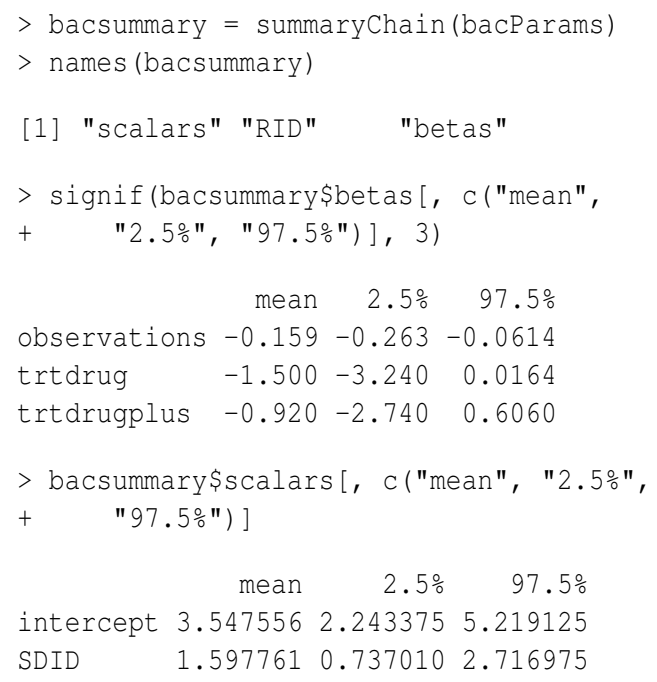

In order to check the convergence of the simulations, we use the checkChain function to produce trace plots of the intercept and $\sigma$ parameters.
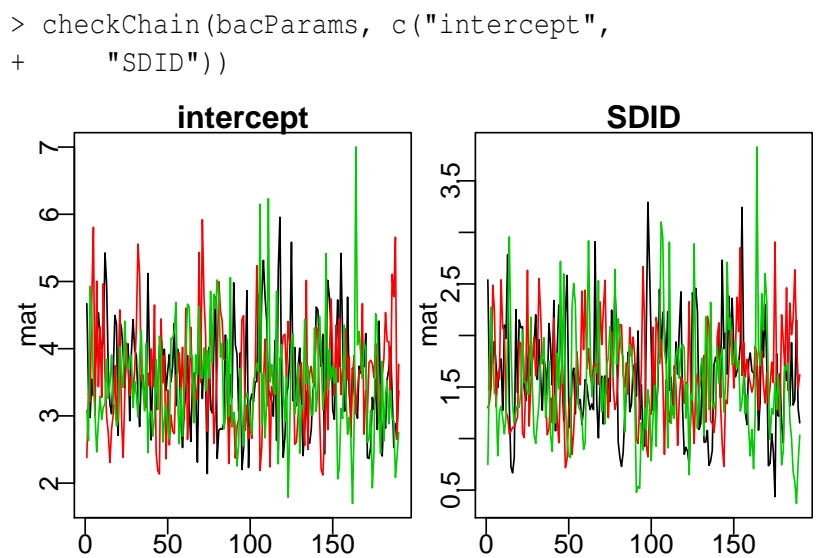


\section{A spatial example}

The ontario dataset contains expected and observed cases of molar cancer by census sub-division in Ontario, Canada.

$>\operatorname{data}($ ontario)

$>\operatorname{head}($ ontario)

$\begin{array}{rrrr} & \text { CSDUID } & \text { observed } & \text { logExpected } \\ 3501005 & 3501005 & 61 & 2.118865 \\ 3501011 & 3501011 & 50 & 1.971265 \\ 3501012 & 3501012 & 117 & 3.396444 \\ 3501020 & 3501020 & 65 & 1.919814 \\ 3501030 & 3501030 & 37 & 1.779957 \\ 3501042 & 3501042 & 16 & 1.182329\end{array}$

Consider the following model (see Rue and Held, 2005):

$$
\begin{aligned}
Y_{i} & \sim \operatorname{Poisson}\left(\lambda_{i} E_{i}\right) \\
\log \left(\lambda_{i}\right) & =\mu+U_{i}+V_{i} \\
U_{i} & \sim \operatorname{GMRF}\left(\sigma_{U}^{2}\right) \\
V_{i} & \sim \operatorname{iid} \mathrm{N}\left(0, \sigma_{V}^{2}\right)
\end{aligned}
$$

where $Y_{i}$ and $E_{i}$ are the observed and expected number of cancer cases in census division $i ; \lambda_{i}$ is the cancer incidence rate; the $U_{i}$ are spatial random effects, a Gaussian Markov random field with variance $\sigma_{U}^{2}$; and $V_{i}$ are spatially independent random effects with variance $\sigma_{V}^{2}$.

This model is reparametrised with $R_{i}=U_{i}+\mu+$ $V_{i}$ in place of $V_{i}$. An adjacency matrix is required for the spatial random effect. This was computed from the spatial boundary files with the poly2nb function from the spdep package and is stored as the object popDataAdjMat. The model can be fitted with glmmBUGS as follows:

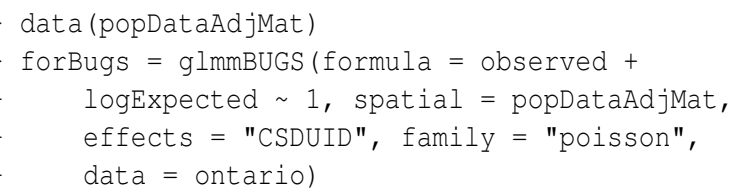

Note that the expected counts are needed on the log scale, and are passed as a second argument on the left side of the model equation to denote their being offset parameters without a coefficient. The random effect is at the census sub-division level (CSDUID), with popDataAdjMat giving the dependency structure. Posterior samples can be generated as follows:

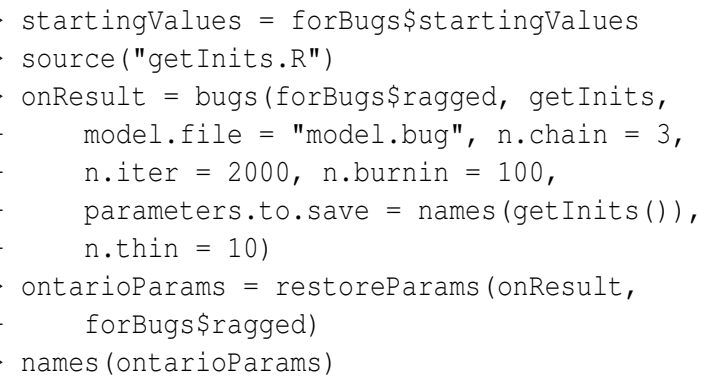

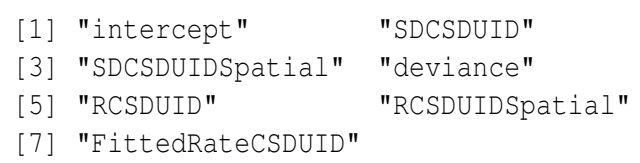

There are posterior simulations for two variance parameters, $\sigma_{U}$ (SDCSDUIDSPATIAL) and $\sigma_{V}$ (SDCSDUID). Samples for the random effects are given for $U_{i}$ (RCSDUIDSpatial), $U_{i}+V_{i}$ (RCSDUID), and $\lambda_{i}$ (FittedRateCSDUID). Trace plots are shown below for $\sigma_{U}$ and $\sigma_{V}$, the standard deviations of the spatial and non-spatial random effects.

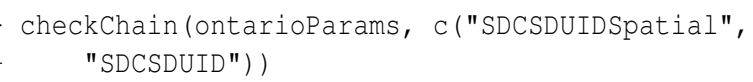

SDCSDUIDSpatial
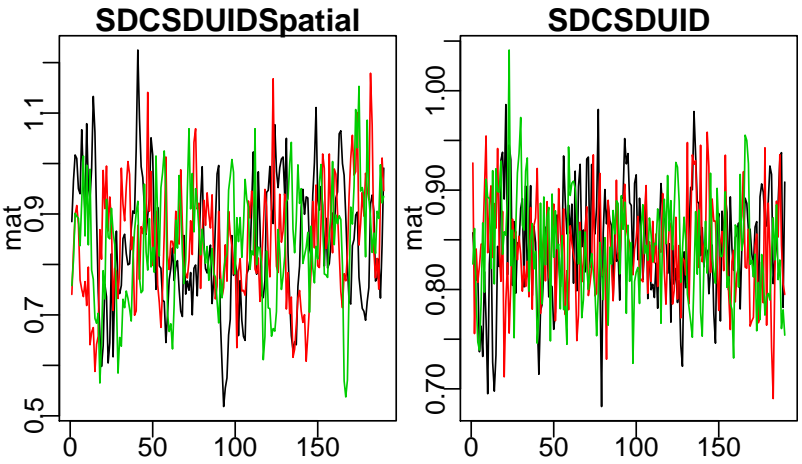

These trace plots show poor mixing, and the Markov chain should be re-run with longer chains and more thinning.

The posterior means for the relative risk $\lambda_{i}$ for Toronto and Ottawa (Census Subdivisions 3506008 and 3530005) are extracted with:

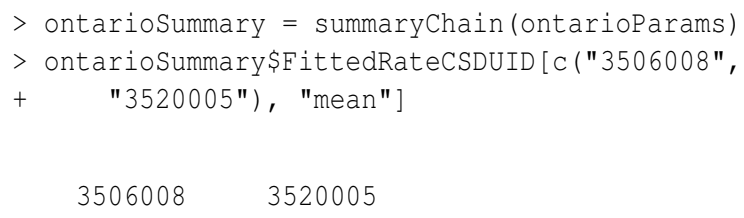

The posterior probability of each region having a relative risk twice the provincial average is computed with

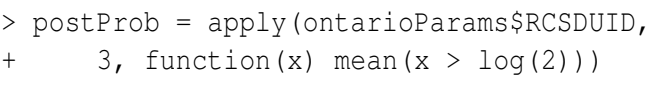

The "RCSDUID" element of ontarioparams is a three dimensional array, with entry $i, j, k$ being iteration $i$ of chain $j$ for region $k$. The apply statement computes the proportion of log-relative risks over $\log (2)$ for each region (the third dimension).

The results can be merged into the original spatial dataset, as described in the documentation for the diseasemapping package. Figure 1 shows a map of the posterior probabilities. 


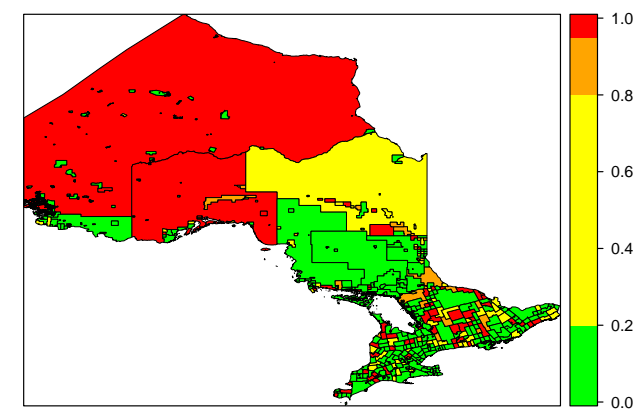

Figure 1: Posterior probabilities of Ontario census sub-divisions having twice the provincial average risk of molar cancer.

\section{Additional features}

The reparam argument to the glmmBUGS function reparametrizes the intercept, and a set of covariates for a new baseline group is provided. Recall that in the bacteria example the ragged object passed to WinBUGS divides covariates into observation and ID level effects. ragged\$Xobservations is a vector and ragged \$XID is a matrix of two columns, which contains the week variable and indicator variables for the treatments respectively. The reparam argument should accordingly be a list with one or more of elements "observations" containing the week or "ID" containing the two treatment indicators. Setting reparam=list (observations $=2$ ) would make week 2 the baseline week, rather than week zero. This argument is useful when using spline functions as covariates, as the intercept parameter which WinBUGS updates can be set to the curve evaluated in the middle of the dataset.

The prefix argument allows a character string to precede all data and variable names. This can be used to combine multiple model files and datasets into a single BUGS model, and modifying these files to allow dependence between the various components. For instance, one random effects model for blood pressure could be given prefix $={ }^{\prime}$ blood', and a model for body mass given prefix $={ }^{\prime}$ mass ${ }^{\prime}$. To allow for dependence between the individual-level random effects for blood pressure and mass, the two data sets and starting values produced by the glmmBUGS function can be combined, and the two model files concatenated and modified. Were it not for the prefix, some variable names would be identical in the two datasets and combining would not be possible.

Finally, multi-level models can be accommodated by passing multiple variable names in the effects argument. If the bacteria data were collected from students nested within classes nested within schools, the corresponding argument would be effects = c ("school", "class", "ID").
Currently unimplemented features that would enhance the package include: random coefficients (specified with an lmer-type syntax); survival distributions (with "Surv" objects as responses); and geostatistical models for the random effects. As WinBUGS incorporates methods for all of the above, these tasks are very feasible and offers of assistance from the community would be gratefully accepted.

\section{Summary}

There are a number of methods and $\mathrm{R}$ packages for performing Bayesian inference on generalized linear mixed models, including geoRglm (Christensen and Ribeiro, 2002), MCMCglmm (Hadfield, 2009) and glmmGibbs (Myles and Clayton, 2001). Particularly noteworthy is the INLA package which avoids the use of MCMC by using Laplace approximations, as described in Rue et al. (2009). Despite this, many researchers working with $\mathrm{R}$ (including the authors) continue to use WinBUGS for model fitting. This is at least partly due to the flexibility of the BUGS language, which gives the user nearly full control over specification of distributions and dependence structures. This package aims to retain this flexibility by providing files to be edited before model fitting is carried out, rather than providing a large number of options for, i.e. prior distributions. More model-specific packages such as INLA are entirely adequate (and perhaps preferable) for the two examples presented here. Those who wish to have full control over priors or build more complex models, for example multivariate or errors-in-covariate models, will likely appreciate the head start which the glmmBUGS package can provide for these tasks.

\section{Acknowledgements}

Patrick Brown holds a Discovery Grant from the Natural Sciences and Engineering Research Council of Canada.

\section{Bibliography}

O. Christensen and P. Ribeiro. geoRglm - a package for generalised linear spatial models. R-NEWS, 2(2):26-28, 2002. URL http://cran.R-project. org/doc/Rnews.

J. D. Hadfield. MCMC methods for multiresponse generalised linear mixed models: The MCMCglmm $\mathrm{R}$ package. URL http://cran.r-project.org/web/packages / MCMCglmm/vignettes/Tutorial.pdf, 2009.

J. Myles and D. Clayton. Package GLMMGibbs. URL http://cran.r-project.org/src/contrib/ Archive/GLMMGibbs, 2002. 
H. Rue and L. Held. Gaussian Markov Random Fields: Theory and Applications, volume 104 of Monographs on Statistics and Applied Probability. Chapman \& Hall, London, 2005.

H. Rue, S. Martino, and N. Chopin. Approximate Bayesian inference for latent Gaussian models by using integrated nested Laplace approximations. Journal of the Royal Statistical Society - Series B, 71 (2):319-392, 2009.

D. Spiegelhalter, A. Thomas, N. Best, and D. Lunn. WinBUGS user manual, 2004. URL http://mathstat.helsinki.fi/openbugs/data/

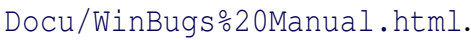

S. Sturtz, U. Ligges, and A. Gelman. R2WinBUGS: A package for running WinBUGS from R. Journal of Statistical Software, 12(3):1-16, 2005. URL http: //www. jstat soft.org.

Patrick Brown

Dalla Lana School of Public Health,

University of Toronto, and

Cancer Care Ontario, Canada

patrick.browneutoronto.ca

Lutong Zhou

Cancer Care Ontario, Canada

carly_zhoudhotmail.com 